\title{
Multihopping Multilevel Clustering Heterogeneity-Sensitive Optimized Routing Protocol for Wireless Sensor Networks
}

\author{
Muhammad Aslam, ${ }^{1}$ Fan Wang, ${ }^{1}$ Xiaopeng Hu, \\ Muhammad Asad, ${ }^{1}$ and Ehsan Ullah Munir ${ }^{2}$ \\ ${ }^{1}$ School of Computer Science and Technology, Dalian University of Technology, Dalian, China \\ ${ }^{2}$ Department of Computer Science, COMSATS Institute of Information Technology, Wah Cantt, Pakistan \\ Correspondence should be addressed to Xiaopeng Hu; xphu@dlut.edu.cn
}

Received 8 February 2017; Revised 24 May 2017; Accepted 12 June 2017; Published 1 August 2017

Academic Editor: Hana Vaisocherova

Copyright (C) 2017 Muhammad Aslam et al. This is an open access article distributed under the Creative Commons Attribution License, which permits unrestricted use, distribution, and reproduction in any medium, provided the original work is properly cited.

Effective utilization of energy resources in Wireless Sensor Networks (WSNs) has become challenging under uncertain distributed cluster-formation and single-hop intercluster communication capabilities. So, sensor nodes are forced to operate at expensive full rate transmission power level continuously during whole network operation. These challenging network environments experience unwanted phenomena of drastic energy consumption and packet drop. In this paper, we propose an adaptive immune Multihopping Multilevel Clustering (MHMLC) protocol that executes a Hybrid Clustering Algorithm (HCA) to perform optimal centralized selection of Cluster-Heads (CHs) within radius of centrally located Base Station (BS) and distributed CHs selection in the rest of network area. HCA of MHMLC also produces optimal intermediate $\mathrm{CHs}$ for intercluster multihop communications that develop heterogeneity-aware economical links. This hybrid cluster-formation facilitates the sensors to function at short range transmission power level that enhances link quality and avoids packet drop. The simulation environments produce fair comparison among proposed MHMLC and existing state-of-the-art routing protocols. Experimental results give significant evidence of better performance of the proposed model in terms of network lifetime, stability period, and data delivery ratio.

\section{Introduction}

Effective environmental monitoring of Wireless Sensor Networks (WSNs) is revolutionized because of vibrant technological advancements of Micro Electromechanical System (MEMS) and wireless communications [1]. Traditionally, WSNs provide successful monitoring of environmental parameters like temperature, pressure, and humidity [2]. Recently, implementation of WSNs has been extended to industrial level in both civil and military applications [3]. The modern tiny sensor devices are intelligent enough to utilize different components of sensing units, data processing units, and transmission techniques that enhance the attractions in WSNs [4]. In given network area, random deployment of sensor nodes is executed along with centralized coordinator called Base Station (BS). BS is responsible for collecting the information from all sensors grouped into clusters. Existing
WSNs are facing technical limitations such as network architecture, short range radio capabilities, and small size initial battery power. So, these limitations challenge researchers to design energy efficient communications protocols to maintain better reliability of network operations [5-7].

Many energy efficient clustering routing protocols have been designed which demonstrate remarkable improvement in prolonging network lifetime. However, issues like reliable cluster-formation, stability, and optimal throughput still need researchers' attention to design optimal solutions [6-9]. LEACH, LEACH-C, SEP, DEEC, HADCC, and so many other protocols gained considerations and were challenged by some other advance techniques [10-16]. Earlier extensive research contains realistic drawbacks of having fixed preprogrammed centralized or distributed cluster-formation algorithms [17-21]. These clustering algorithms lack in online hybrid workability at runtime, along with multihopping 
data delivery under shadow of hybrid cluster-formation. To the best of our knowledge there is no hybrid clusterformation routing protocol that can support multihopping. Therefore, we propose a unique and novel routing protocol called Multihopping Multilevel Clustering (MHMLC) using Hybrid Clustering Algorithm (HCA) cluster-formation. The objective and contributions of our proposed model are as follows:

(1) MHMLC brings reliable environmental sensing and reporting due to reduction in average packets delay and path loss that enabled proposed model to achieve better packet delivery ratio with minimum latency. This MHMLC's achievement is mainly because of introducing unique hybrid HCA algorithm. HCA maintains less complex centralized cluster-formation and single-hop reporting in nearby region of BS, as well as maintaining distributed cluster-formation in remote region with a distinguished multihop transmission capabilities. Single-hop communications in nearby hot-spot region result in reduction of delay and path loss because of limited transmission range, while multihop communication in far region from BS makes sure of less packet drop and avoiding packet retransmissions that result in higher packet delivery ratio.

(2) MHMLC provides better stability and network lifetime due to coordination of hybrid cluster-formation and even distribution of traffic responsibilities over all sensor nodes according to their residual energy resources and distance from BS. In this way, MHMLC establishes intelligent networking that makes sure of having enough resistance to avoid early departure of nodes that increase the stability and network lifetime.

(3) MHMLC offers better realistic opportunities to be deployed in real-time practical environment because of flexible cluster-formation that is designed according to the nature of sensor nodes basic capabilities.

(4) Experimental results show that MHMLC produces $127 \%, 109 \%, 91 \%, 58 \%$, and $5 \%$ higher stability period than LEACH, SEP, LEACH-C, DEEC, and HADCC protocols, respectively. Furthermore, the proposed model has less end-to-end delay and path loss and enhances packets delivery ratio as compared to wellknown routing protocols.

The rest of this paper is organized as follows. Section 2 contains related work and characteristics comparison, Section 3 contains detailed network design and system model, Section 4 deals with the proposed model, Section 5 represents results with analytical discussion, and Section 6 concludes this paper.

\section{Related Work and Motivation}

The initial considerable success of WSNs increases the industrial demand of these networks to provide additional challenging features. WSNs need improvements at every level such as sensor's vendor, initial battery capacity, economical sensing, information processing, and energy efficient reporting to BS. During transmission, routing algorithms are utilized to adopt the most energy efficient routes between sensor nodes and BS. Routing protocols contain path-planning algorithms, which are primarily responsible for effective data delivery to central controller of BS [1-9]. In study of different clustering protocols, some novel techniques are investigated to understand their performance during network operations in WSNs. Some related state-of-the-art protocols are discussed in this section.

In [10], LEACH introduced a dynamic distributed approach which runs complete probabilistic algorithm to nominate $\mathrm{CHs}$, which accept the responsibilities of association phase to complete cluster's membership. This process avoids supervision of BS; in this case nodes bear all computational and communication cost. $\mathrm{CH}$ s collect data from member nodes at single-hop transmissions and perform aggregated transmission to BS. This distributed algorithm brings fluctuation in $\mathrm{CH}$ selection that causes significant performance degradation.

In [11], LEACH-C tried to mitigate uncertain fluctuations in $\mathrm{CHs}$ creations, by introducing centralized algorithm in which BS is primarily responsible for nomination of $\mathrm{CHs}$. BS utilized nodes energy level and location attributes to set priority of nodes in order to lead the member nodes. However, entire centralized cluster-formation for whole network region demands extensive additional transmissions over the long distance that results in unwanted energy dissipation. Moreover, LEACH and LEACH-C are designed for homogeneous networking environments and lack practicality in heterogeneous WSNs.

In [12], SEP proposed two-level clustering for heterogeneous WSNs. In SEP, nodes with higher energy are referred to as advanced nodes which have more probability for selection than CHs. SEP also ignores the realistic overall heterogeneity of the network and it is unable to mitigate issues of fully distributed $\mathrm{CH}$ s selections.

In [13], DEEC proposal represents efficient clustering within multiheterogeneous network environment, in which residual energy of each node is responsible for its probability index. However, in complete distributed cluster-formation, nodes have fewer opportunities to share the global knowledge in the whole network. In DEEC, average numbers of CHs per round are much more as compared to other protocols, which brings additional direct transmissions towards BS which dissipate more energy resources.

In [14], a hybrid model has been proposed with the name of HADCC; the major contribution to establish this protocol is to handle region-wise homogeneity and heterogeneity intelligently. Another contribution of this model is to establish an algorithm, which can be centralized and distributed at the same time, and both properties can be helpful in different regions of the networks. However, HADCC only produce single-hop intercluster communication that is real drawback of its performance.

Major portion of existing routing protocols are unable to address the issues of network scalability and load balancing because of single-hop routes constructions, although 
TABLE 1: Comparison of the state-of-the-art clustering routing protocols.

\begin{tabular}{|c|c|c|c|c|c|}
\hline Features & MHMLC & LEACH & CEEC & DEEC, SEP, ESEP & MLEACH \\
\hline Heterogeneity-aware & $\sqrt{ }$ & & & $\sqrt{ }$ & \\
\hline Extendable & $\sqrt{ }$ & & & $\sqrt{ }$ & $\sqrt{ }$ \\
\hline Manageable & $\sqrt{ }$ & & $\sqrt{ }$ & & \\
\hline Distance-based design & $\sqrt{ }$ & & $\sqrt{ }$ & & \\
\hline Avoiding sudden dissipation & $\sqrt{ }$ & & $\sqrt{ }$ & & $\sqrt{ }$ \\
\hline Coverage of whole network & $\sqrt{ }$ & & $\sqrt{ }$ & & $\sqrt{ }$ \\
\hline Controlled CHs formation & $\sqrt{ }$ & & $\sqrt{ }$ & & \\
\hline Controller assistance & $\sqrt{ }$ & & & & $\sqrt{ }$ \\
\hline Reducing complexity & $\sqrt{ }$ & $\sqrt{ }$ & & & \\
\hline Network support & $\sqrt{ }$ & $\sqrt{ }$ & & & \\
\hline Online reconfiguration & $\sqrt{ }$ & $\sqrt{ }$ & & & \\
\hline Energy efficiency & $\sqrt{ }$ & & & & \\
\hline Hierarchal forwarding & $\sqrt{ }$ & & & & $\sqrt{ }$ \\
\hline Avoiding redundant transmissions & $\sqrt{ }$ & $\sqrt{ }$ & $\sqrt{ }$ & & $\sqrt{ }$ \\
\hline Application-aware & $\sqrt{ }$ & & $\sqrt{ }$ & $\sqrt{ }$ & \\
\hline Higher data delivery & $\sqrt{ }$ & & & & $\sqrt{ }$ \\
\hline Cooperative routing for long range transmission & $\sqrt{ }$ & & & $\sqrt{ }$ & $\sqrt{ }$ \\
\hline
\end{tabular}

these less complex routing protocols are helpful in quick path discovery with minimum resource utilization and need minimum computational complexity but also struggle when having to perform link rediscovery in long complex network. Furthermore, these algorithms are not efficient to extend the network capacity. In order to handle these issues multihopping algorithms are proposed, which try to fulfill the high performance demands and keep establishing network paths under all conditions. Multihop routing protocols are supposed to generate and regenerate efficient multiple paths with minimum packet drop and reduce energy dissipation. However, existing protocols need more sophisticated algorithms to achieve the established goals [21-27].

In this paper, we propose a routing protocol called Multihopping Multilevel Clustering (MHMLC) energy efficient path-planning routing protocol supported by versatile HCA, which provides not only suitable hybrid $\mathrm{CHs}$ selection but also unique multihopping ability. These abilities of MHMLC bring better stability and throughput performance at simulations level and also bring better performance in practical environment. Characteristics comparison of MHMLC with state-of-the-art routing protocol is given in Table 1.

\section{System Network Design Problem Statement}

The key factors of attention to construct better sensor network's design are network modeling, node's reporting schedule, data aggregation technique, and radio model characteristics. Our proposed work is based on well-defined problem statement and system design to achieve the aforementioned major objectives. List of extensively used abbreviations is given in Notations.

3.1. System Design. Initially whole network region is assumed as two-dimensional rectangular area with $A$ to $B$ limits at $x$ axis and $C$ to $D$ limits at $y$-axis. So, network area $[A, B] \times$
$[C, D]$ is divided into $i$ clusters along $x$-axis and $j$ along $y$ axis. Every cluster position $\left(x_{i}, y_{j}\right)$ represents an independent subarea. BS can compute height of surface, $f\left(x_{i}, y_{j}\right)$, and the Average Surface Height (ASH) of all clusters is approximately equal to height of whole network, which can be calculated as

ASH

$$
=\frac{f\left(x_{o}, y_{o}\right)+\cdots+f\left(x_{1}, y_{1}\right)+\cdots+f\left(x_{i-1}, y_{j-1}\right)}{I \times J} .
$$

Approximation sigma notation can be used to generalize this scalability:

$$
\mathrm{ASH} \approx \frac{1}{I} \sum_{i=0}^{I-1} \sum_{j=0}^{J-1} f\left(x_{i}, y_{j}\right),
$$

where total length of $i$ and $j$ can be defined as $I=B-A$ and $J=D-C$. From this, ASH scalability approximation can be derived as

$$
\mathrm{ASH} \approx \frac{1}{[B-A][D-C]} \sum_{i=0}^{I-1} \sum_{j=0}^{J-1} f\left(x_{i}, y_{j}\right) \Delta x \Delta y,
$$

where $\Delta x$ and $\Delta y$ are variation at $i$ and $j$ and special notation for the limit of double sum can be defined as

$$
\mathrm{ASH} \approx \lim _{I J \rightarrow \infty} \sum_{i=0}^{I-1} \sum_{j=0}^{J-1} f\left(x_{i}, y_{j}\right) \Delta x \Delta y .
$$

In this way, double integration optimization can be derived from the above expressions:

$$
\begin{aligned}
& \lim _{I J \rightarrow \infty} \sum_{i=0}^{I-1} \sum_{j=0}^{J-1} f\left(x_{i}, y_{j}\right) \Delta x \Delta y=\int_{A}^{B} \int_{C}^{D} f\left(x_{i}, y_{j}\right) d A \\
& \text { ASH }=\lim _{I J \rightarrow \infty} \int_{A}^{B} \int_{C}^{D} f\left(x_{i}, y_{j}\right) d A .
\end{aligned}
$$




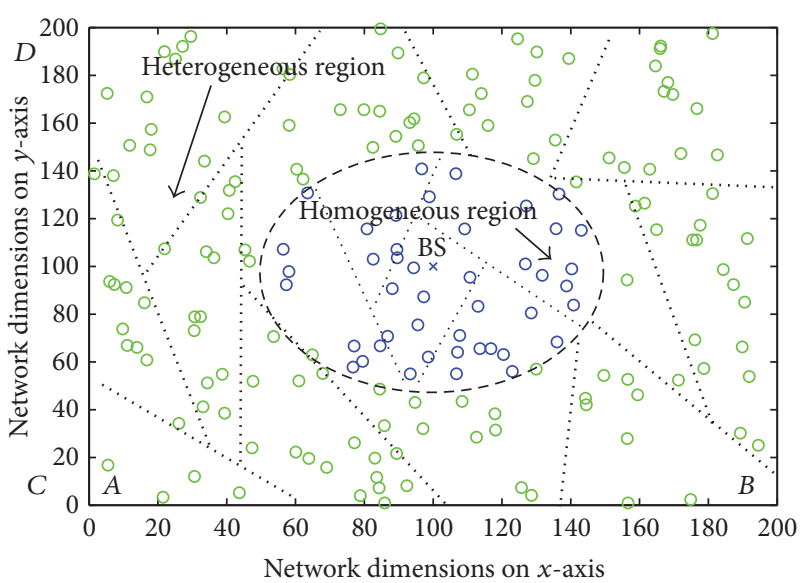

Figure 1: Network model to execute MHMLC's cluster-formation.

Furthermore, BS is located in the center of the network and maintains a logical inner-homogeneous region within a specific radius; outer region is considered as heterogeneous portion as shown in Figure 1.

3.2. Energy Model for Radio Characteristics. First-order linear radio model is preferred for proposed scheme's radio characteristics in order to observe the performance of MHMLC, as it has been examined for other well-known conventional protocols [10-15]. First-order linear radio model defines 50 nJ/bit energy consumption for transceiver circuitry, denoted by $E_{\text {eleRX }}$ and $E_{\text {eleTX}}$. Acceptable $E_{b} / N_{0}$ restricts certain transmission amplification parameters and is shown as $E_{\mathrm{amp}}$. Energy consumption linear model that ignores the sensing and computational cost is represented as

$$
E_{\mathrm{TX}}(L, d)= \begin{cases}L \times E_{\text {elec }}+S \times E_{\mathrm{fs}} d^{2} & \text { If } d<d_{o} \\ L \times E_{\text {elec }}+S \times E_{\mathrm{amp}} d^{4} & \text { If } d \geq d_{o}\end{cases}
$$

Total energy consumption $\left(E_{\mathrm{TX}}(L, d)\right)$ can be calculated in reference of free space and multipath models with $E_{\mathrm{fs}} d^{2}$ and $E_{\text {amp }} d^{4}$, respectively. $S$ is data size transmitted over distance of $d$ while $d_{o}$ is reference distance over which selection of free space and multipath model is selected.

3.3. Problem Statement. Efficiency of MHMLC's proposal depends upon the efficient selection of $\mathrm{CHs}$ and non-CHs nodes. In this paper we propose a clustering algorithm which produces very intelligent clustering according to the remaining resources with their historical energy consumption rate and services as $\mathrm{CHs}$. Now the $\mathrm{CHs}$ selected in both regions should be equal to the desired percentage $P$ set by the network administrator which is $10 \%$ in most of the cases [1-5]. Total Desired Percentage of $\mathrm{CHs}$ (TDPCHs) can be calculated as

$$
\mathrm{TDPCHs}=\sum_{i=1}^{N \times P_{\mathrm{DLNs}}} \mathrm{CHs}_{\text {Level1 }}+\sum_{j=1}^{N \times P_{\text {CLNs }}} \mathrm{CHs}_{\text {Level2 } 2}
$$

Basic Threshold-based $\mathrm{CH}$ (TCH) selection criterion of $\mathrm{CHs}_{\text {Level1 }}$ is threshold value of Distributed Level Nodes (DLNs), which is calculated by

$\mathrm{T}(\mathrm{CH})$

$$
= \begin{cases}\frac{P_{l}}{1-P_{l} *\left(r \bmod \left(1 / P_{l}\right)\right) \times d} & \text { if } \mathrm{DLN}_{l} \in G r^{\prime} \\ 0 & \text { otherwise. }\end{cases}
$$

Ideally $P$ for DLNs should meet a certain threshold value to become CHs; similarly BS sets priorities of Centralized Level Nodes (CLNs) to select $\mathrm{CHs}_{\text {Level2 }}$; this calculation is carried by

$$
\text { Epoch-ECR }=\int_{r=0}^{R_{\text {epoch }}} p(t) d t .
$$

The details of (8) and (9) are given in the next section.

\section{Proposal of Multihopping Multilevel Clustering Energy Efficient Protocol}

MHMLC is mainly proposed to establish an energy efficient clustering routing protocol that improves the highly demanded QoS parameters of reliability, with better network lifetime and higher data delivery. MHMLC enforces clusterformation through Hybrid Clustering Algorithm (HCA) which is primarily responsible for updating nodes ID and their regional sector ID. Furthermore distance-based threshold regional ID assigned by HCA assists nodes to participate in centralized or distributed Cluster-Heads (CHs) selection. MHMLC operation is divided into iterative periods called rounds; furthermore each round is subdivided into multiple phases. The main structural phases of HCA-based wellequipped MHMLC approach are as follows.

4.1. Regional Dimensions Preparation Phase. The total number of $N$ scattered sensors experiences initialization in network region of $M \times M$ (boundaries of $[A, B] \times[C, D]$ ) by the BS. Initially all nodes utilize basic clustering criteria distributively by threshold formula of HCA to calculate their distance from BS. Nodes, within specific radius of centrally located BS, upgrade their regional ID as Centralized Level Nodes (CLNs); similarly outsider nodes upgrade their regional ID as Distributed Level Nodes (DLNs):

$$
\mathrm{NDL}= \begin{cases}\text { Centralized Level Nodes } & \text { if dist } \leq \mathrm{rL} \\ \text { Distributed Level Nodes } & \text { if dist } \geq \mathrm{rL},\end{cases}
$$

where NDL is Nodes Distance Level and $\mathrm{rL}$ is radius Level of BS. In mobility based scenario NDL gradation will occur in every round but in static environment nodes just need initial gradation that will be considered during whole network operation. Nodes upgraded to DLNs suddenly proceed with distributive HCA algorithm property; otherwise they wait for BS to initialize centralized HCA selection of CHs. Transmission of events that occurred demands $\mathrm{K}$ representative $\mathrm{CHs}$, and each $\mathrm{CH}$ accommodates set of member nodes (MNs) in its cluster. 
4.2. Distributed Property of HCA's CHs Selection. HCA starts cognitive selection of optimum number of $\mathrm{CH}$ in heterogenous distributed environment. DLNs are made locationaware through GPS installed equipment, able to memorize initial energy level $\left(E_{O}\right)$, with computational ability to calculate their residual energy level $\left(E_{r}\right)$, and Energy Consumption Rate (ECR). In heterogeneous environment these values play tie breaker role for HCA to select the most suitable $\mathrm{CHs}$. Nodes initialize exchanging hello packets with $\left(E_{O}, E_{r}, \mathrm{ECR}\right)$ within tier-one level neighbors. Initial hello packets contain timer $T 1$ field that allows single-hop propagation; after that it will decrease to $T 0$, so second-tier level neighbors will be saved from overhearing effect.

In distributed CHs selection, all nodes have their election weight; ideally in case of homogeneous environment every node $\mathrm{DLN}_{l}$ (where $l=1,2, \ldots, L$ ) has equal probability of $1 / P_{\text {opt }}$, to become $\mathrm{CH}$ over specific period of epoch. In heterogeneous environment, every $\mathrm{DLN}_{l}$ has different responsibility to serve as $\mathrm{CH}$ that depends upon its $E_{r}$, while, over a single period, a set of $\mathrm{CHs}_{k}(k=1,2, \ldots, K)$ serves as leading nodes to transmit their respective cluster report. In heterogeneous network, nodes with higher weights have better chance to become CHs, so their epoch size will also be small. Average residual energy of DLNs will be

$$
\bar{E}(r)=\frac{1}{L} \sum_{l=1}^{L} E_{r}
$$

Similarly total energy of all $\mathrm{DLN}_{l}$ is calculated to measure CH's selection probability of heterogeneous $\mathrm{DLN}_{l}$ :

$$
E_{\text {toat }}=\sum_{l=1}^{L} E_{O}(1+\alpha) \text {, }
$$

where $\alpha$ represents extra energy factor of higher energy nodes, while optimal amount of CHs is prescribed as

$$
P_{l}=P_{\text {opt }} \frac{E_{l}}{\bar{E}(r)} \text {. }
$$

Equation (13) indicates the fact that, in heterogeneous environment, optimal number of $\mathrm{CH}$ depends upon the remaining energy resources. DLNs participate in probabilistic election to declare themselves as $\mathrm{CHs}$. Each node individually calculates a threshold with the following equation:

$\mathrm{T}(\mathrm{CH})$

$$
= \begin{cases}\frac{P_{l}}{1-P_{l} *\left(r \bmod \left(1 / P_{l}\right)\right) \times d} & \text { if } \mathrm{DLN}_{l} \in G r^{\prime} \\ 0 & \text { otherwise. }\end{cases}
$$

In this equation, $\mathrm{T}(\mathrm{CH})$ is calculation of threshold, computed independently on every node $\mathrm{DLN}_{l}, G r$ is group of nodes which are competing in this distributed election of current round, and $d$ is distance from BS. This distance factor increases the chance of nodes closer to BS to be selected as $\mathrm{CH}$. This selection helps to keep data transmissions towards BS, which efficiently avoids backward transmissions. Now every node $\mathrm{DLN}_{l}$ generates a random number and compares its value to $\mathrm{T}(\mathrm{CH})$. If $\mathrm{T}(\mathrm{CH})$ is greater than random number; then it will be selected as potential $\mathrm{CH}$; otherwise it will automatically consider itself as member node.

Heterogeneity-awareness establishes the fact that nodes with different energy maintain different probability levels, so probability is calculated as

$$
P_{\text {normal }}=\frac{P_{\mathrm{opt}}}{1+\alpha m}
$$

where $P_{\text {normal }}$ is probability of normal node (lower energy level node); similarly probability of advance nodes (higher energy nodes) can be calculated as

$$
P_{\text {advance }}=\frac{P_{\mathrm{opt}}(1+\alpha)}{1+\alpha m} \text {. }
$$

In similar fashion, value of $P_{l}$ can be calculated for two-level heterogeneity:

$$
P_{l}= \begin{cases}\frac{P_{\mathrm{opt}} E_{l}(r)}{(1+\alpha m) \bar{E}(r)} & \text { If node is normal } \\ \frac{P_{\mathrm{opt}}(1+\alpha) E_{l}(r)}{(1+\alpha m) \bar{E}(r)} & \text { If node is Advance. }\end{cases}
$$

For multilevel heterogeneous network $P_{l}$ can be calculated as

$$
P_{S_{l}}=\frac{P_{\mathrm{opt}} L\left(1+\alpha_{l}\right)}{L+\sum_{l=1}^{L} \alpha_{l}} .
$$

The above equations indicate that the higher residual energy and the smaller distance of $\mathrm{DLN}_{l}$ from $\mathrm{BS}$ enhance the probability of them to be elected as $\mathrm{CHs}$. Nodes also generate the last threshold to break the epoch rounds if it is getting longer to terminate the traffic flow from the same $\mathrm{CH}$ s that will distribute burden evenly; epoch threshold is calculated as

$$
\text { Epoch }= \begin{cases}\text { Continue } & \text { if Epoch } \leq 10 \\ \text { Terminate } & \text { if Epoch }>10\end{cases}
$$

4.3. Centralized Property of HCA's CHs Selection. Simultaneously, $\mathrm{CLN}_{m}$ within central region waits for BS to initialize cluster-formation. Central property of hybrid HCA executes few computational works to calculate Energy Consumption Ratio (ECR) for each $\mathrm{CLN}_{m}$ :

$$
\operatorname{ECR}(m)=\frac{E_{0}}{E_{0}-E_{r}} .
$$

After calculating ECR, HCA calculates their Residual Energy Ratio (RER):

$$
\operatorname{RER}(m)=\frac{E_{r}}{\mathrm{ECR} \times d_{\mathrm{toBS}}},
$$


where $d_{\text {toBS }}$ is distance of $\mathrm{CLN}_{m}$ from BS. If we add ECR equation, the RER will be

$$
\operatorname{RER}(m)=\frac{E_{r}}{E_{0} /\left(E_{0}-E_{r}\right) \times d_{\mathrm{toBS}}} .
$$

After calculation of ECR and RER for current round, HCA calculates the integrated value of nodes' ECR and RER over certain period of epoch. As energy consumption rate is power $p=E_{r} / T$, HCA utilizes Riemann sum for power dissipated over time. This ECR over time period of epoch can be calculated as approximation:

$$
\text { Epoch-ECR } \approx \sum_{r=0}^{R_{\text {epoch }}} p\left(t_{i}\right) \Delta t_{i}
$$

where Epoch-ECR is ECR of whole epoch. Integral form of this energy consumption over epoch period will be

$$
\text { Epoch-ECR }=\int_{r=0}^{R_{\text {epoch }}} p(t) d t .
$$

Similarly, HCA computes the RER of previous epoch with the above equations; in this way BS selects set of suitable nodes with minimum energy consumption and maximum residual energy. Distance factor also plays tie breaker rule to select a $\mathrm{CLN}$ as $\mathrm{CH}$ node for central region node. The pseudocode of hybrid CHs selection of HCA is shown in Algorithm 1.

These following work-steps represent the $\mathrm{CHs}$ section procedure indicated by the above HCA algorithm.

Step 1. Initially, location-awareness of nodes distributes nodes into CLNs and DLNs.

Step 2. DLNs calculate respective probability of being selected as CHs.

Step 2.1. DLNs calculate threshold values.

Step 2.2. DLNs generate random number and compare it with threshold value.

Step 2.3. If the random number is less than threshold, nodes select themselves as $\mathrm{CHs}$.

Step 3. BS computes ECR for CLNs.

Step 3.1. BS computes Epoch-ECR and then selects a set of potential CHs.

Step 3.2. BS finally selects $P \times$ CLNs as $\mathrm{CHs}$ for centralized region.

4.4. Association Phase of MHMLC. Successfully elected CHs in both regions start broadcasting in neighborhood by exchanging hello packets. CHs switch their radio mode from sleep to active and remain active during the whole round until they perform as $\mathrm{CHs}$, while non-CHs are allowed to stay in sleep mode after certain reporting time. Non-CHs receive multiple advertisements from the neighborhood and

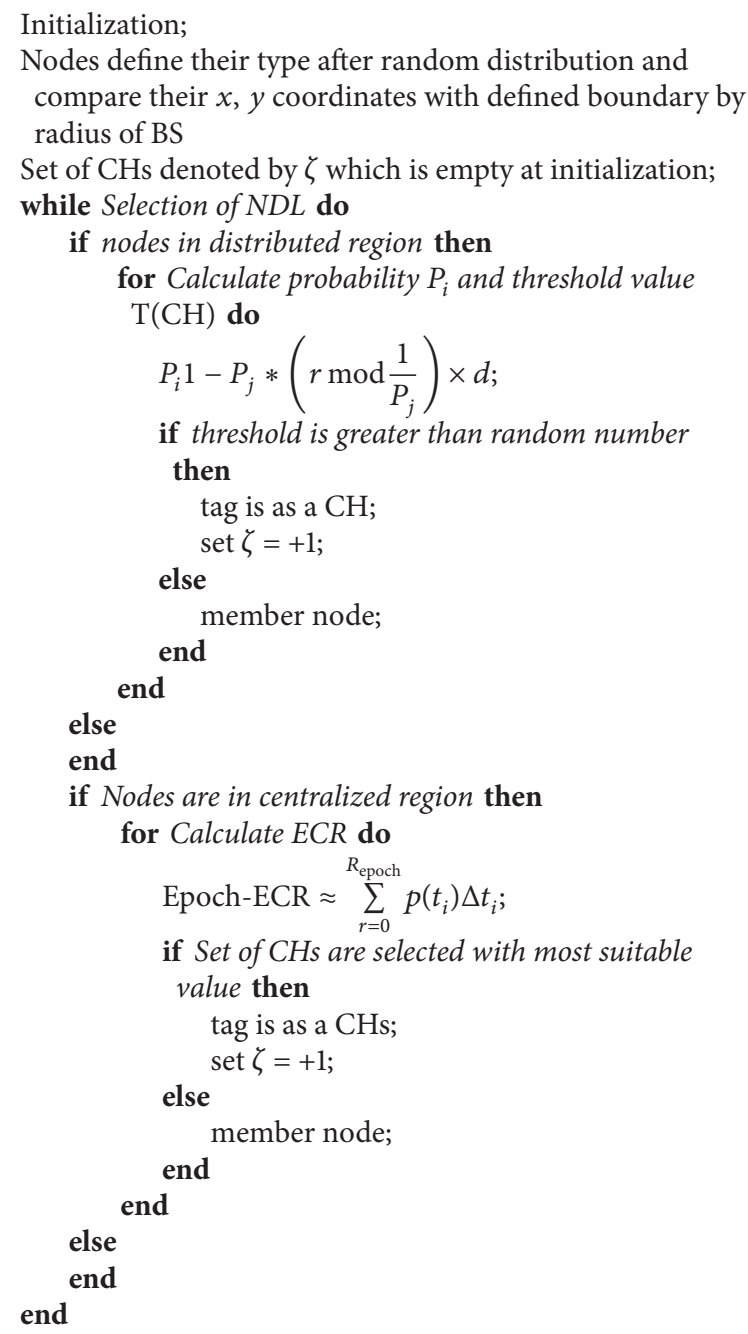

Algorithm 1: Cluster-Heads selection by HCA.

then calculate the association criteria to associate with best $\mathrm{CH}$.

$$
\text { Association }_{\text {criteria }}=\frac{\mathrm{RSSI}+\mathrm{RER}}{\mathrm{Dis}_{\text {InRangeCH }}},
$$

where RSSI is Received Signal Strength Indication (RSSI) that is major factor in deciding link quality to minimize packet drop ratio and path loss. In order to avoid collision and packet drop, non-CHs generate association request using CSMA MAC protocol, while CHs keep waiting to receive the final decision of nodes. As neighbor nodes send their association preference, $\mathrm{CHs}$ finalize cluster-formation and start multicasting TDMA slots; in this way member nodes can transmit sensed reports within specific scheduling.

4.5. Network Transmission Phase of MHMLC. Transmission phase represents the actual communication of environmental reports from the network field. MHMLC adopts outstanding unique multihopping in order to achieve the goal of 
energy efficiency, reliability, and minimum delay transmission. For intracluster communication, MHMLC adopts short range single-hop transmissions, while multihopping is implemented for intercluster communications. In this technique, multiple links are created by availability of intermediate $\mathrm{CH}$ that accept communicative neighborhood that offer maximum reliability with minimum link energy-budget. Initially all selected $\mathrm{CH}$ react as only source node for associated clusters and initiate route discovery. In this process, $\mathrm{CH}$ transmit route-request including demanded criteria for intermediate $\mathrm{CHs}$ and in response receive available multipath IDs that construct possibility index for source $\mathrm{CH}$ and intermediate $\mathrm{CHs}$. This route discovery process enables intermediate $\mathrm{CHs}$ to elect best next-hop neighboring $\mathrm{CH}$ s towards the BS. All these processes create rating index of available multipaths and $\mathrm{CH}$ select the best one based on highest ranking. This ranking function delivers like a proxy server in order to select a suitable neighbor $\mathrm{CH}$. Source $\mathrm{CH}$ scores intermediate $\mathrm{CHs}$ by the ratio of cluster load $C_{L}$ and service time $S_{T}$. The theoretical ideal ranking the $\mathrm{CHs}$ can be calculated by

$$
R_{0}=\frac{C_{L}}{S_{T}}
$$

But in realistic implementation there are so many factors playing their part to affect scoring mechanism among which calculation delay $D_{C}$ and uncertainty weight $U_{W}$ are the most significant factors. Now more realistic scoring function of a neighbor $\mathrm{CH}$ can be calculated:

$$
S_{F}=\frac{C_{L}+D_{i}+U w_{i}}{S_{T}},
$$

where $S_{F}$ is the scoring function. This linear scoring ranking of neighbor $\mathrm{CH}$ can mislead all source $\mathrm{CH}$ to fight for the most fast neighbor with lowest load and quickest response time as the above equation provides conclusive evidence. To tackle this greedy algorithmic nature source $\mathrm{CH}$ includes another factor of distance between source $\mathrm{CH}$ and possible neighbor $\mathrm{CH}$ in range. This neighbor realistic $S_{F}$ selection can be calculated by following equation:

$$
S_{F}^{\prime}=\frac{C_{L}+D_{i}+U w_{i}}{S_{T}+D_{N}},
$$

where $D_{N}$ is distance towards neighbor CHs. The above equation just provides flexibility in order to select neighbor $\mathrm{CHs}$ as intermediate CHs. For further enhancement, HCA restricts intermediate $\mathrm{CHs}$ selection, by set of threshold values. This threshold-based selecting ability of intermediate $\mathrm{CHs}$ can be calculated as

$$
\mathrm{T}(\mathrm{NCH})= \begin{cases}S_{F}^{\prime} & \text { if } k \in N K \\ 0 & \text { otherwise }\end{cases}
$$

where $\mathrm{T}(\mathrm{NCH})$ is threshold value to select the intermediate $\mathrm{CHs}$, one specific neighbor from $k$ is the available $N K$
TABLE 2: Simulation parameters.

\begin{tabular}{lc}
\hline Deployed sensors & 100 \\
Initial network dimensions & $100 \mathrm{~m} \times 100 \mathrm{~m}$ \\
Optimal percentile of required CHs & $10 \%$ \\
Information aggregation dissipation & $50 \mathrm{pj} / \mathrm{bit}$ \\
Packet size & $4000 \mathrm{bit}$ \\
Transmitter electronics circuit & $50 \mathrm{nj} / \mathrm{bit}$ \\
Receiver electronics circuit & $50 \mathrm{nj} / \mathrm{bit}$ \\
Transmitter amplifier energy dissipation & $100 \mathrm{pj} / \mathrm{bit} / \mathrm{m}^{2}$ \\
\hline
\end{tabular}

neighbor CHs pool; if we put the value of $S_{F}^{\prime}$, then we get the following:

$$
\mathrm{T}(\mathrm{NCH})= \begin{cases}\frac{C_{L}+D_{i}+U w_{i}}{S_{T}+D_{N}} & \text { if } k \in N K \\ 0 & \text { otherwise. }\end{cases}
$$

Now source CHs can utilize these scoring functions threshold values to create effective intermediate $\mathrm{CH}$ rate selection that will help all intermediate $\mathrm{CH}$ sot to drain energy due to repeated selection. These thresholds can be applied to provide further more controlled load balancing in the system. This load balancing also indicates that less tested intermediate $\mathrm{CH}$, although having slow processing capability, provides availability to source CHs. In this load balancing multihopping technique we can achieve the reliability and low delay transmission that can achieve better energy efficiency to prolong the network lifetime. Flow chart of proposed MHMLC can be observed in Figure 2.

\section{Simulation Results and Discussion}

This section evaluates the performance analysis over simulations experiments of MHMLC and its compared protocols. MATLAB is used to simulate existing protocols along with of MHMLC. Simulative results under conventional setting have been observed in order to maintain the realistic outcomes. Random distribution of nodes is initialized in specific settings. Homogeneous sensors are scattered within specific radius of centralized BS, while heterogeneous nodes are scattered outside centralized region. Initial clustering operation of MHMLC test is shown in Figure 3. Moreover, radius size, number of nodes, and whole network size have been modified extensively in order to judge the performance in different scenario. Initial network parameters and linear radio model's specifications are given in Table 2.

Performance of MHMLC has been critically analyzed after testing experiments in presence of existing state of the art routing protocols like SEP, DEEC, LEACH, LEACH-C, and HADCC.

The following performance measurement criteria are usually considered vital for critical analysis.

(1) Network Lifetime. Network lifetime is prescribed by the total duration of network operation until last node is alive. 


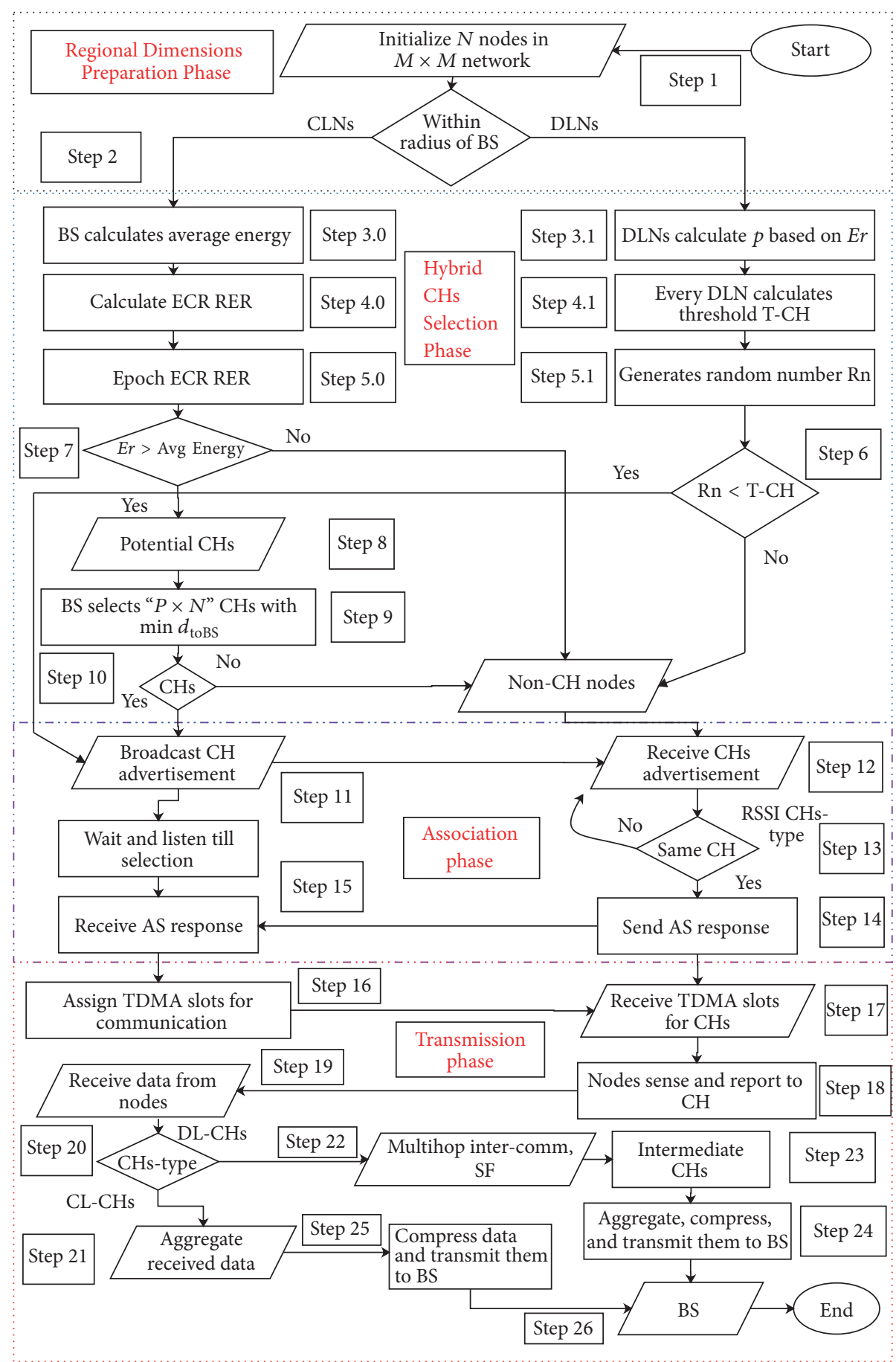

FIgURE 2: Flow chart of MHMLC mechanism.

(2) Stability Period. It is the duration of network operation over which all nodes are alive and it ends with expiry of first node in the network. Stability is a key factor that describes the performance of overall network throughput.

(3) Instability Period. When nodes begin to die, instability period is started; during this period network faces drastic packet drop.
(4) Selection of $\mathrm{CHs}$. It indicates the number of $\mathrm{CH}$ senerated per round. Periodic operation experiences fluctuation in selection of $\mathrm{CH}$ in every round. This fluctuation determines expected data delivery ratio and path loss experienced by WSNs.

(5) Packet to BS. This shows the amount of data successfully received by $\mathrm{BS}$ from $\mathrm{CHs}$. 


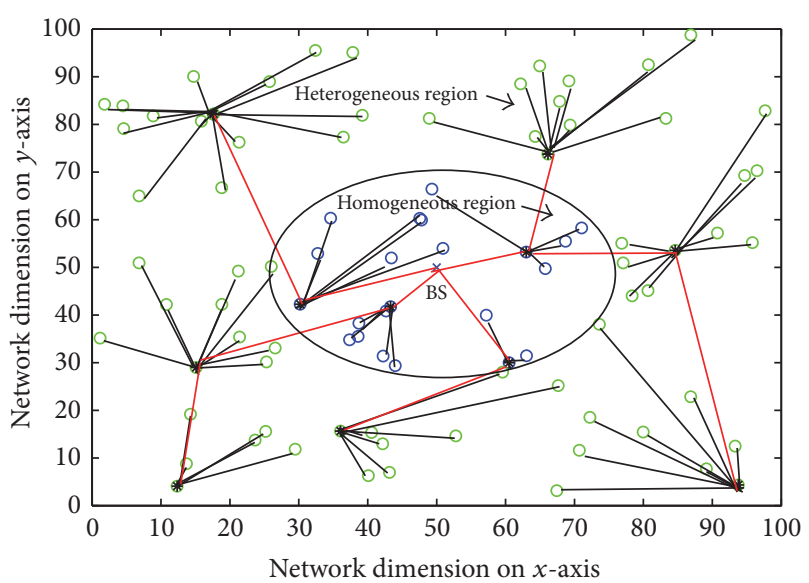

Figure 3: Clustering topology in MHMLC.

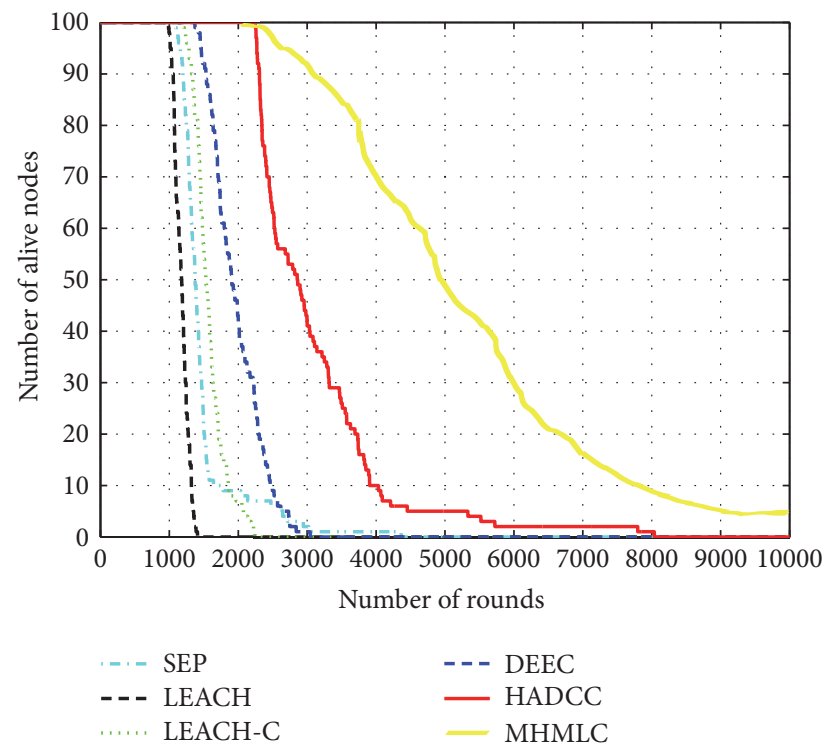

Figure 4: Lifetime and stability period for alive nodes.

5.1. Overall Network Operational Period and Stability Period. Figure 4 shows stability period and overall network lifetime of competed protocols in initial network scenario of $100 \mathrm{~m}$ $\times 100 \mathrm{~m}$ with 100 nodes. This experiment indicates that MHMLC attains $127 \%, 109 \%, 91 \%, 58 \%$, and $5 \%$ better stability period than LEACH, SEP, LEACH-C, DEEC, and HADCC protocols, respectively. More significantly network lifetime of MHMLC is almost 9, 8, and 3 times better than $\mathrm{LEACH}, \mathrm{LEACH}-\mathrm{C}$, and DEEC, respectively, while, in case of HADCC, it shows $51 \%$ betterment. Figure 5 shows that the resistance during instable period in case of LEACH, LEACH$\mathrm{C}$, SEP, and DEEC nodes experiences sudden expiration after first node death and HADCC produces some fight but its single-hop transmissions cause early exit of alive nodes. However, MHMLC design is flexible enough that distributes traffic burden intelligently over $\mathrm{CHs}$ and attains higher instable period along with stability period and network life time.

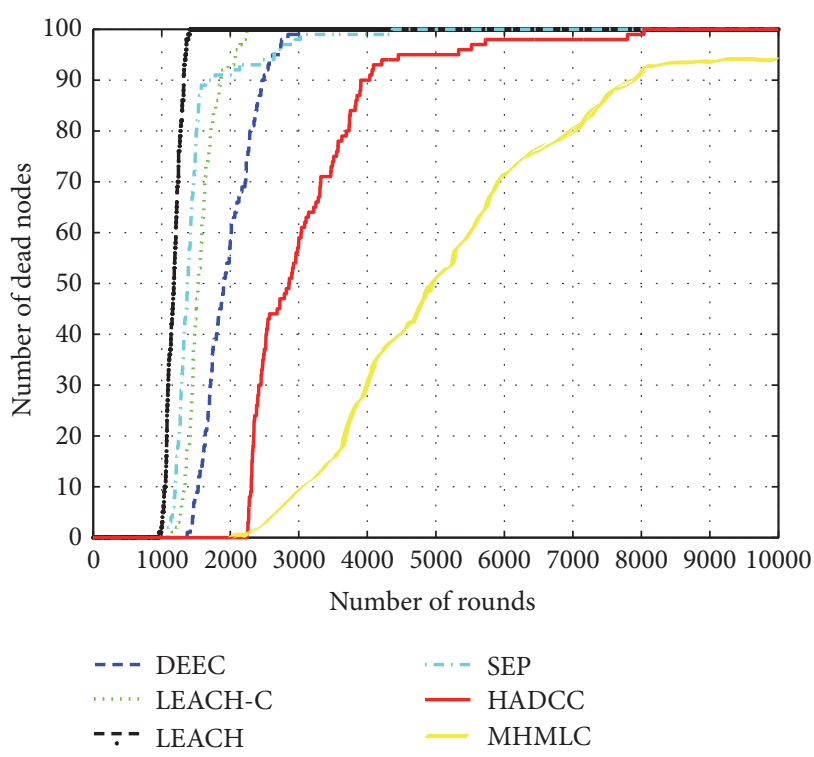

FIGURE 5: Resistance with respect to number of dead nodes.

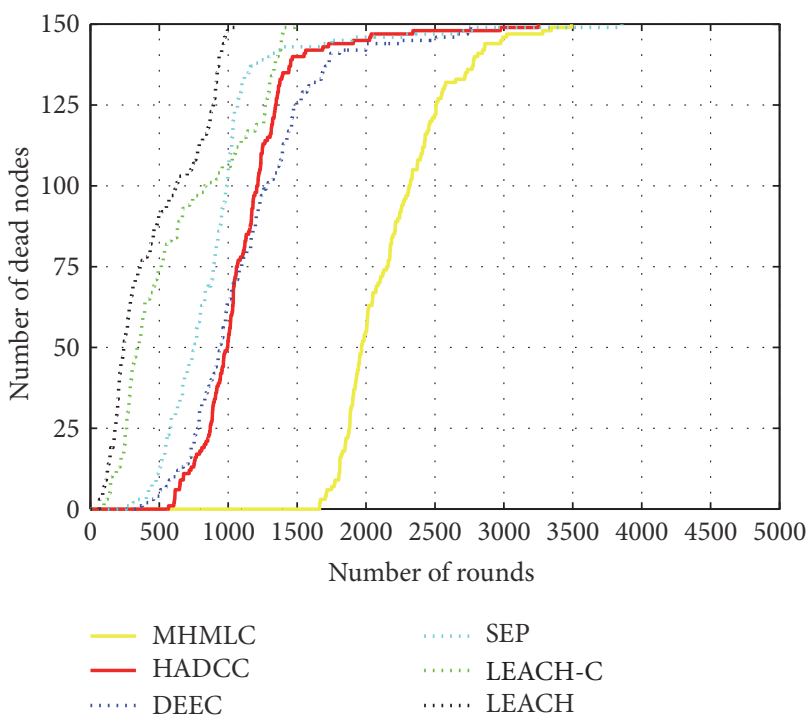

FIGURE 6: Network lifetime in $300 \mathrm{~m} \times 300 \mathrm{~m}$ network area with 150 nodes.

Figures 6 and 7 indicate performances measurements over network areas $300 \mathrm{~m} \times 300 \mathrm{~m}$ and $500 \mathrm{~m} \times 500 \mathrm{~m}$, respectively. $300 \mathrm{~m} \times 300 \mathrm{~m}$ and $500 \mathrm{~m} \times 500 \mathrm{~m}$ network areas contain 150 nodes and 200 nodes, respectively. Figures 6 and 7 provide clear indications of degradation of all competed protocols as network area and network density get increased, where, in both cases, MHMLC make sure of having better stability and network life time as compared to other protocols. Furthermore, we extended simulation-based experiment to $700 \mathrm{~m} \times 700 \mathrm{~m}$ and $1000 \mathrm{~m} \times 1000 \mathrm{~m}$. Overall stability period can be analyzed in Figure 8. As network scalability and density increase, the multihopping ability of MHMLC becomes more relevant factor of improvement. 


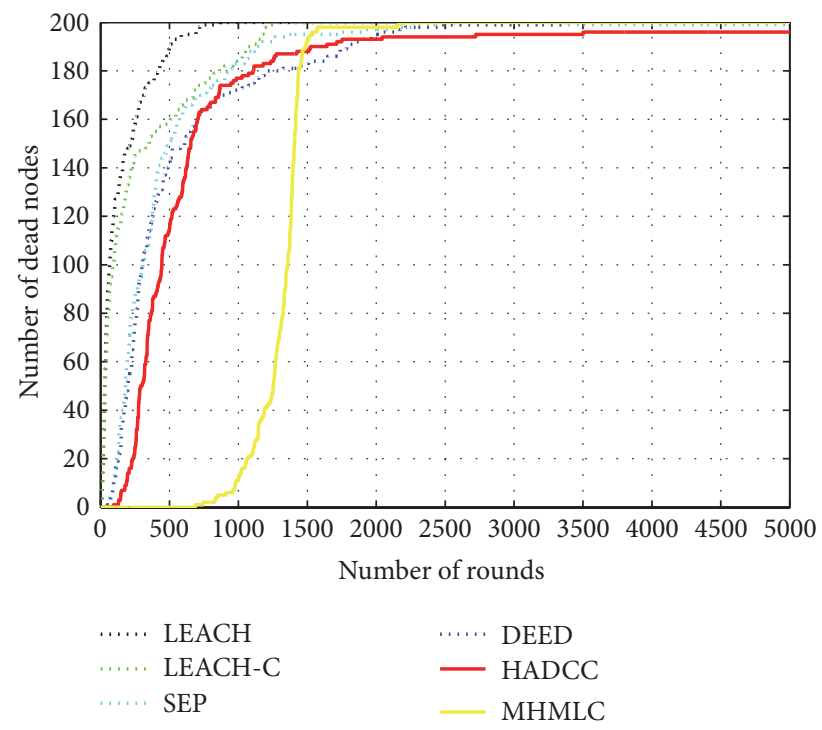

FIGURE 7: Network lifetime in $500 \mathrm{~m} \times 500 \mathrm{~m}$ network area with 200 nodes.

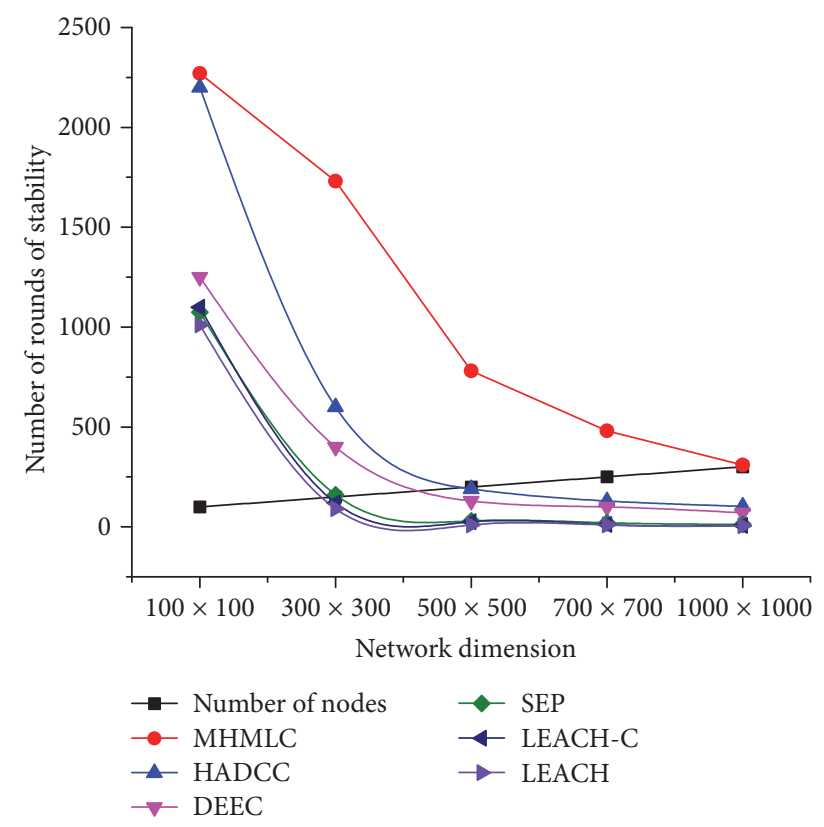

FIGURE 8: Network stability with multiple heterogeneity.

5.2. Efficient Network Settling and Transmission Phase. In Figure 9 outcomes indicate achieved performance of throughput results which validate the maximum data delivery in case of MHMLC, while other protocols like DEEC and HADCC have some competency but in the long term these protocols face higher path loss. SF-based multihop capability of MHMLC enables the sustainable intercluster communication that provides resistance to maintain link connectivity during transmissions phase. These characteristics of MHMLC result in higher data delivery as path-fault tolerance and reconfiguration abilities have been featured in proposed model. These abilities minimize the possibility of fatal packet drops and

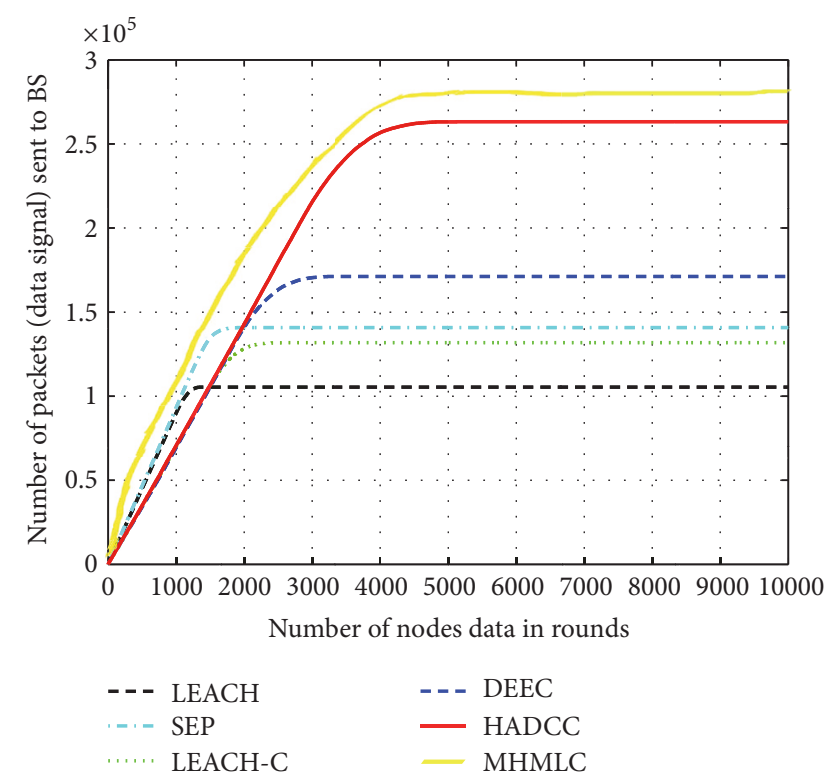

FIgURE 9: Amount of data transmitted to BS.

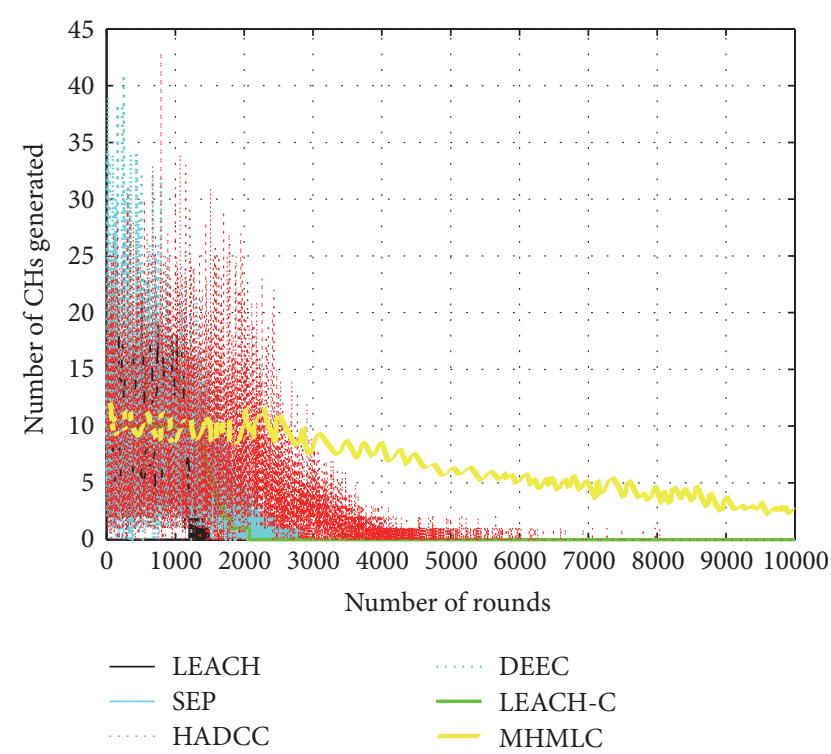

FIGURE 10: CHs generations over the round periods.

enable MHMLC protocol to produce better throughput as compared to other protocols.

In Figure 10, another important phenomenon has been illustrated in the process of $\mathrm{CHs}$ generations during the operation network. Conventional distributed protocols of LEACH, SEP, and DEEC cause drastic fluctuations up to $1-33 \%$ CHs, while HCA makes sure of the selection of optimal $\mathrm{CHs}$ and minimization of fluctuations to $8-12 \%$. Initial efficient intracluster $\mathrm{CH}$ of hybrid HCA algorithm continue till selection of intermediate $\mathrm{CH}$ for multihop intercluster communications. Figure 11 indicates higher fluctuation of CHs selection in extended network area of $300 \mathrm{~m} \times 300 \mathrm{~m}$ with 150 nodes, which reflects the fact that cluster-formation 


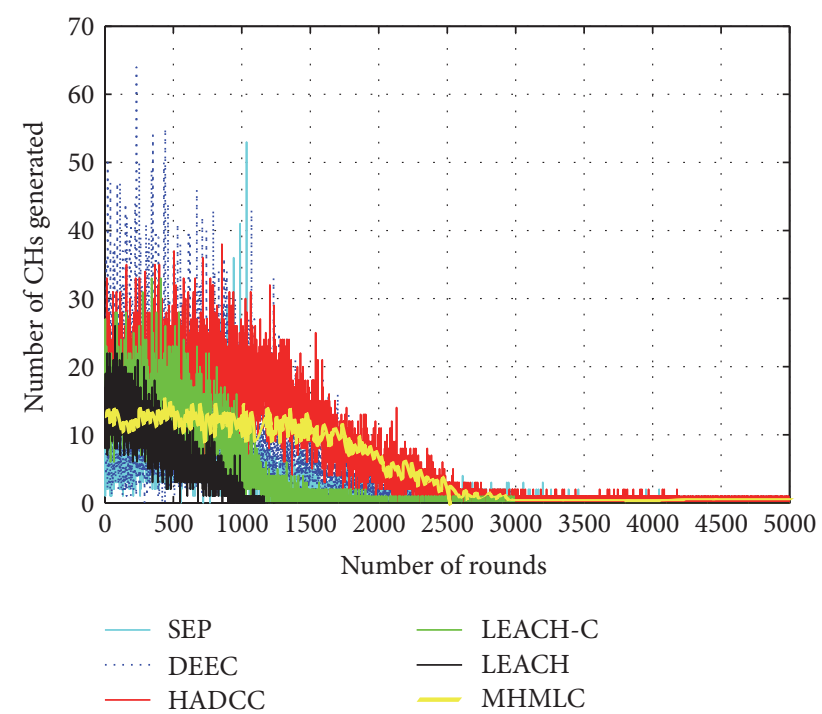

FIgURE 11: Number of CHs generated in $300 \mathrm{~m} \times 300 \mathrm{~m}$ area with 150 nodes.

is more challenging in bigger network. These higher fluctuations bring down the performance of fully centralized or distributed protocols; in this scenario hybrid clusterformation of MHMLC becomes more relevant and provides better performance than compared protocols.

5.3. Packet Delivery Ratio, Path Loss, and End-to-End Delay. In conventional settings, ideal assumptions define the optimistic transmission model. This optimistic model knowingly ignores the signal distortions effects like interference, fading, and signal attenuation. Furthermore, BS unfolds the enveloped reports with $100 \%$ success without any consideration of sampling error rate. But in real network scenario, these challenges occur and need careful probabilistic measurement to calculate more actual efficiency. Current performance evolution adopts uniform random distribution model that determines packet drop $\left(P_{d}\right)$ probability. $P_{d}$ changes dynamically according to the network mobility pattern and distance between sensor nodes and BS. Link quality plays tie breaker role and always demanded to be high to compete with $P_{d}$ for better packets delivery. Deterministic value of $P_{d}$ is as follows:

$$
P_{\mathrm{DSH}}= \begin{cases}0, & \text { if } 0 \leq \text { dist } \leq 30 \\ \left(\frac{1}{70}\right) \times(\text { dist }-30) & \text { if } 30 \leq \text { dist } \leq 100 \\ 1, & \text { if dist }>100 .\end{cases}
$$

$P_{\mathrm{DSH}}$ is packet drop probability in single-hop intercluster communication conditions. $P_{d}$ in multihop intercluster communications will be less drastic as $\mathrm{CHs}$ use short range intermediate $\mathrm{CHs}$, and it can be expressed as

$$
P_{\mathrm{DMH}}= \begin{cases}0, & \text { if } 0 \leq \text { dist } \leq 30 \\ \left(\frac{1}{95}\right) \times(\text { dist }-30) & \text { if } 30 \leq \text { dist } \leq 100 \\ 1, & \text { if dist }>100 .\end{cases}
$$

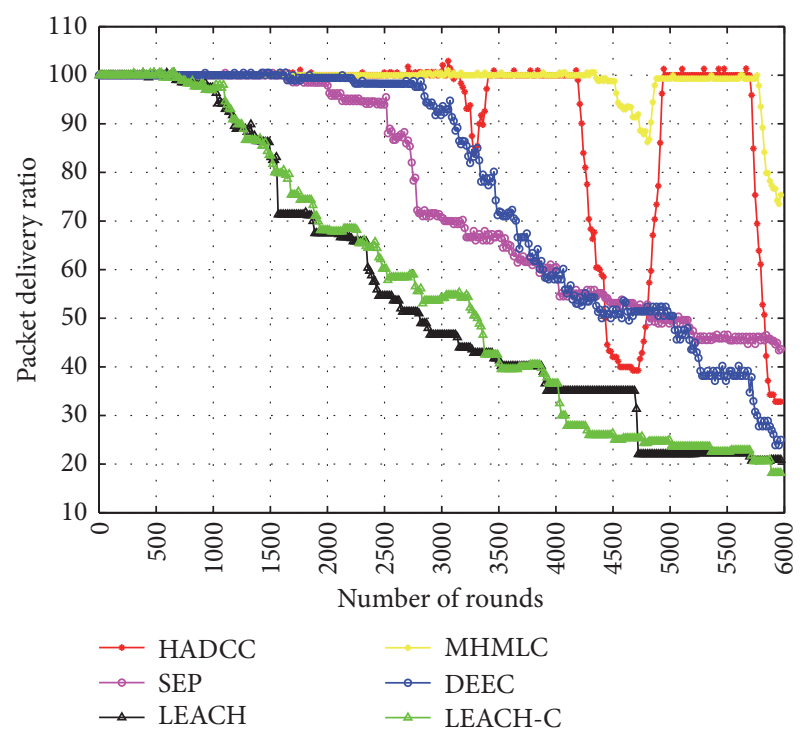

FIGURE 12: Packet delivery ratio over the operational rounds.

In order to measure the packet drop ratio deeply in simulation environment, we increase initial energy level of all nodes to get better observations over longer period network operation. Figure 12 shows that packet delivery ratio of MHMLC is way better than other compared protocols. A major reason behind this development advocates the fact that nodes start getting drained out and lesser nodes remain alive, which causes unwanted phenomena of higher $P_{\mathrm{DSH}}$ as compared to multihop $P_{\mathrm{DMH}}$. Similarly path loss of distributed routing models is much higher than MHMLC as shown in Figure 13. MHMLC maintains the better connectivity and utilizes short distance radio links intelligently. Figure 14 indicates the end-to-end delay faced by tested protocols, and this result indicates the lower delay in case of proposed model. This delay reduction is due to less complex HCA's central selection of $\mathrm{CHs}$ in nearby region of $\mathrm{BS}$, which later on adopts direct transmissions to BS.

5.4. Path Loss and Packet Delivery Ratio of Proposed Model in Different Scenario. We simulated MHMLC extensively, with respect to different scenarios; to represent better picture we selected four scenarios of network model of $100 \mathrm{~m} \times$ $100 \mathrm{~m}, 1500 \mathrm{~m} \times 150 \mathrm{~m}, 170 \mathrm{~m} \times 170 \mathrm{~m}$, and $200 \mathrm{~m} \times 200 \mathrm{~m}$. But in every scenario we extended node's initial energy in order to study the data transmission metrics. Figures 15 and 16 show the performance measurements of data delivery ratio and path loss for MHMLC with different network size. These figures depict that as network size increases, the performance decrement occurs significantly. This also highlights the encouraging fact that MHMLC resists well and makes it possible to keep data delivery ratio acceptable in bigger network execution. Figure 17 indicates the fact that the larger number of alive nodes will result in more complex cluster-formation. This causes redundant transmission paths that add overhead to make routing decisions. So initially we notice significant delay when network is too crowded but with 


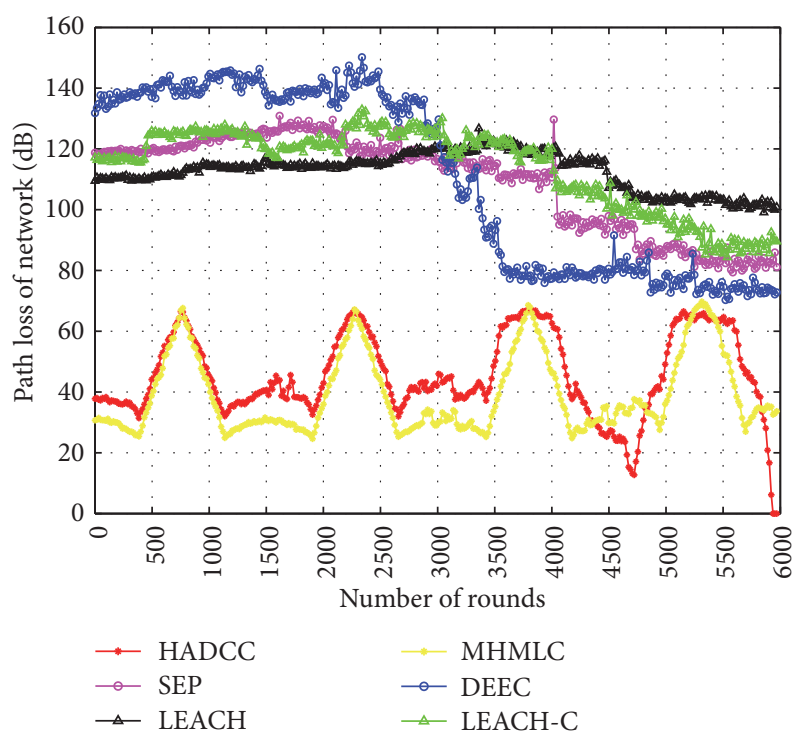

Figure 13: Path loss over network operational rounds.

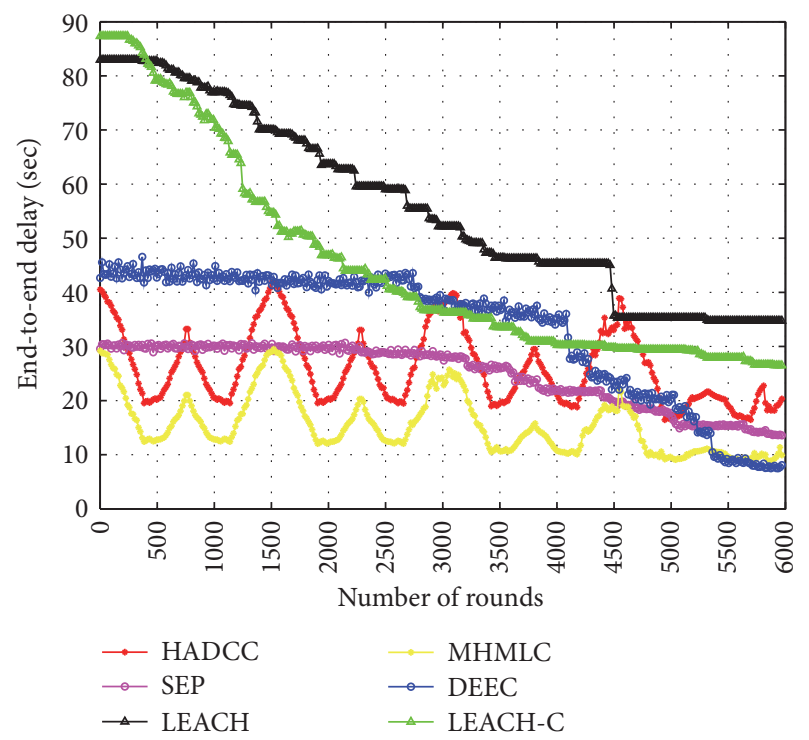

FIGURE 14: End-to-end delay over network operation.

network operation as density goes low, the routing complexity starts getting reduced and minimizes the delay.

\section{Conclusion and Future Work}

This paper proposes heterogeneity-sensitive routing protocol called MHMLC, with novel Hybrid Clustering Algorithm (HCA) that contributes to efficient $\mathrm{CHs}$ selection. HCA adds flexibility in MHMLC with introducing reliable clusterformation. HCA adopts partially distributed $\mathrm{CHs}$ selection heterogeneous region and partial central $\mathrm{CHs}$ selection central homogeneous region with coordination of BS. Another significant improvement of MHMLC is its unique multihopping support during interclustering communication. Obtained performance analysis of simulations results with

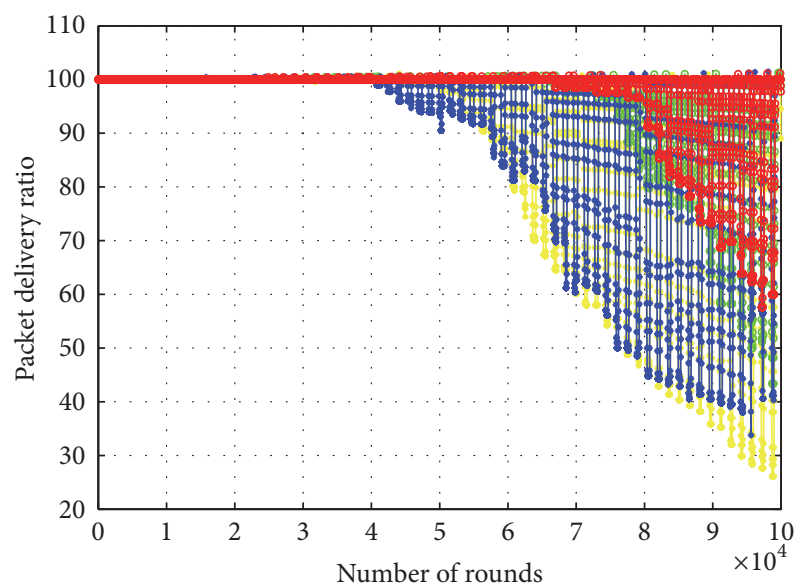

- MHMLC exectution in network size of $200 \mathrm{~m} * 200 \mathrm{~m}$

$-\quad$ MHMLC exectution in network size of $150 \mathrm{~m} * 150 \mathrm{~m}$

$\rightarrow \quad$ MHMLC exectution in network size of $170 \mathrm{~m} * 170 \mathrm{~m}$

$\rightarrow$ MHMLC exectution in network size of $100 \mathrm{~m} * 100 \mathrm{~m}$

FIGURE 15: Packet delivery ratio in different network size.

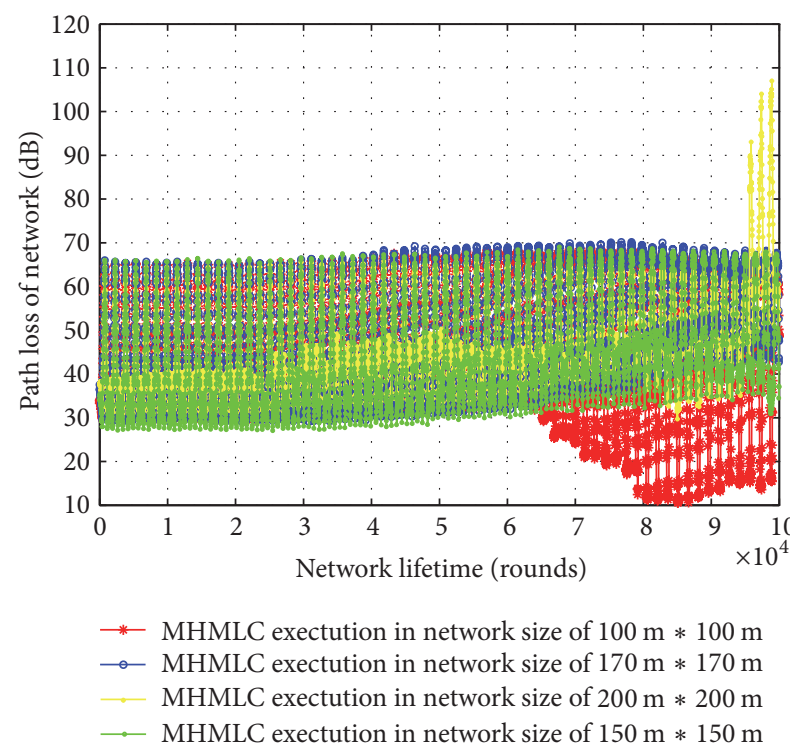

Figure 16: Path loss experienced in different network size.

multiple conventional routing protocols encourage our proposed model, as MHMLC outperforms all other protocols. Experimental results validate our theoretical proposal of MHMLC and indicate significant developments that result in better network lifetime, stability, and data delivery for WSNs.

In this paper, we test our proposal in simulations environment; further research on practical implementation and its performance betterment in extraordinary large network can be fruitful.

\section{Notations}

HCA: Hybrid Clustering Algorithm

NDL: Nodes Distance Level 


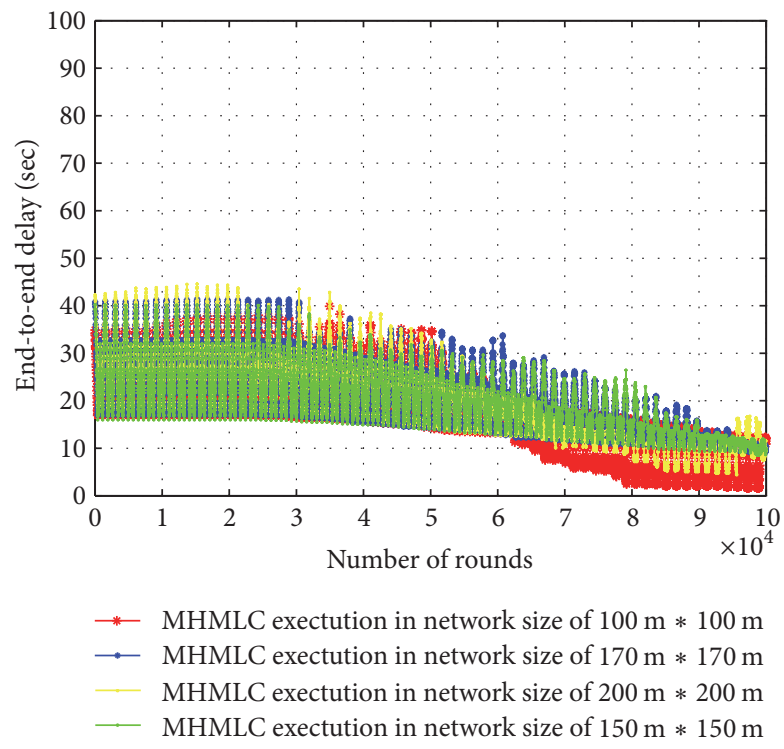

FIGURE 17: End-to-end delay for over different network size.

DLN: Distributed Level Nodes

CLN: Centralized Level Nodes

ASH: Average Surface Height

TDPCH: Total Desired Percentage of Cluster-Heads

$P_{\text {normal }}: \quad$ Probability of normal nodes

$P_{\text {advance: }}$ Probability of advance nodes

RER: $\quad$ Residual Energy Ratio

$C_{L}: \quad$ Cluster load

$S_{T}: \quad$ Service time

$C_{D}: \quad$ Calculation delay

$U_{W}: \quad$ Uncertainty weight

$S_{F}: \quad$ Significant factor

$\mathrm{T}(\mathrm{NCH})$ : Threshold value of Neighbor $\mathrm{CH}$

$E_{\text {elec }}: \quad$ Transceiver energy

$E_{\mathrm{TX}}(L, d)$ : Total energy consumption

$E_{\text {eleRX}}$ : Circuitry Receiving Energy

$E_{\text {eleTX }}$ : Circuitry Transmitting Energy

$P_{\text {DLNs }}: \quad$ Desired percentage of DLNs

$P_{\mathrm{DLNs}}: \quad$ Desired percentage of CLNs.

\section{Conflicts of Interest}

The authors declare that there are no conflicts of interest regarding the publication of this paper.

\section{Acknowledgments}

This research is supported in part by National Natural Science Foundation of China (NSFC) (no. 61272523, no. 61471084) and the Fundamental Research Funds for the Central Universities.

\section{References}

[1] F. Wang, S. Wu, K. Wang, and X. Hu, "Energy-efficient clustering using correlation and random update based on data change rate for wireless sensor networks," IEEE Sensors Journal, vol. 16, no. 13, pp. 5471-5480, 2016.

[2] N. A. Pantazis, S. A. Nikolidakis, and D. D. Vergados, "Energyefficient routing protocols in wireless sensor networks: a survey," IEEE Communications Surveys and Tutorials, vol. 15, no. 2, pp. 551-591, 2013.

[3] T. van Dam and K. Langendoen, "An adaptive energy-efficient MAC protocol for wireless sensor networks," in Proceedings of the 1st International Conference on Embedded Networked Sensor Systems (SenSys '03), pp. 171-180, New York, NY, USA, November 2003.

[4] N. Javaid, M. Aslam, K. Djouani, Z. A. Khan, and T. A. Alghamdi, "ATCEEC: a new energy efficient routing protocol for wireless sensor networks," in Proceedings of the 2014 1st IEEE International Conference on Communications (ICC '14), pp. 263268, June 2014.

[5] M. Yu, K. K. Leung, and A. Malvankar, "A dynamic clustering and energy efficient routing technique for sensor networks," IEEE Transactions on Wireless Communications, vol. 6, no. 8, pp. 3069-3079, 2007.

[6] Y. Min, Y.-K. Kim, and J.-W. Chang, "An energy-efficient routing protocol using message success rate in wireless sensor networks," Journal of Convergence, vol. 4, pp. 15-22, 2013.

[7] B. Yahya and J. Ben-Othman, "REER: robust and energy efficient multipath routing protocol for wireless sensor networks," in Proceedings of IEEE Global Telecommunications Conference (GLOBECOM '09), pp. 1-7, Honolulu, HI, USA, December 2009.

[8] Y. Yao, Q. Cao, and A. V. Vasilakos, "EDAL: an energy-efficient, delay-aware, and lifetime-balancing data collection protocol for heterogeneous wireless sensor networks," IEEE/ACM Transactions on Networking, vol. 23, no. 3, pp. 810-823, 2015.

[9] F. Wang, Y. Yang, K. Wang, X. Hu, and N. Zhang, "Efficient clustering routing algorithm based on opportunistic routing," International Journal of Communications, Network and System Sciences, vol. 09, no. 05, pp. 198-208, 2016.

[10] W. R. Heinzelman, A. Chandrakasan, and H. Balakrishnan, "Energy-efficient communication protocol for wireless microsensor networks," in Proceedings of the 33rd Annual Hawaii International Conference on System Siences (HICSS '00), p. 10, January 2000.

[11] W. B. Heinzelman, A. P. Chandrakasan, and H. Balakrishnan, "An application-specific protocol architecture for wireless microsensor networks," IEEE Transactions on Wireless Communications, vol. 1, no. 4, pp. 660-670, 2002.

[12] G. Smaragdakis, I. Matta, and A. Bestavros, "SEP: a stable election protocol for clustered heterogeneous wireless sensor networks," Tech. Rep., Boston University Computer Science Department, 2004.

[13] L. Qing, Q. Zhu, and M. Wang, "Design of a distributed energyefficient clustering algorithm for heterogeneous wireless sensor networks," Computer Communications, vol. 29, no. 12, pp. 22302237, 2006.

[14] M. Aslam, E. U. Munir, M. Bilal et al., "HADCC: hybrid advanced distributed and centralized clustering path planning algorithm for WSNs," in Proceedings of the 28th IEEE International Conference on Advanced Information Networking and Applications (IEEE AINA '14), pp. 657-664, May 2014.

[15] D. Guo, J. Cao, X. Wang, Q. Fu, and Q. Li, "Combating QRcode-based compromised accounts in mobile social networks," Sensors (Switzerland), vol. 16, no. 9, article no. 1522, 2016.

[16] A. M. S. Saleh, B. M. Ali, M. F. A. Rasid, and A. Ismail, "A self-optimizing scheme for energy balanced routing in wireless 
sensor networks using sensorAnt," Sensors, vol. 12, no. 8, pp. 11307-11333, 2012.

[17] A. Tufail, A. Qamar, A. M. Khan, W. A. Baig, and K.-H. Kim, "WEAMR - a weighted energy aware multipath reliable routing mechanism for hotline-based WSNs," Sensors (Switzerland), vol. 13, no. 5, pp. 6295-6318, 2013.

[18] M. Hammoudeh and R. Newman, "Adaptive routing in wireless sensor networks: QoS optimisation for enhanced application performance," Information Fusion, vol. 22, pp. 3-15, 2015.

[19] C.-H. Lung and C. Zhou, "Using hierarchical agglomerative clustering in wireless sensor networks: an energy-efficient and flexible approach," Ad Hoc Networks, vol. 8, no. 3, pp. 328-344, 2010.

[20] J. Luo, J. Hu, D. Wu, and R. Li, “Opportunistic routing algorithm for relay node selection in wireless sensor networks," IEEE Transactions on Industrial Informatics, vol. 11, no. 1, pp. 112-121, 2015.

[21] E. P. De Freitas, T. Heimfarth, C. E. Pereira, A. M. Ferreira, F. R. Wagner, and T. Larsson, "Evaluation of coordination strategies for heterogeneous sensor networks aiming at surveillance applications," in Proceedings of the IEEE Sensors 2009 Conference (SENSORS '09), pp. 591-596, October 2009.

[22] A. Aijaz and A. H. Aghvami, "Cognitive machine-to-machine communications for internet-of-things: a protocol stack perspective," IEEE Internet of Things Journal, vol. 2, no. 2, pp. 103112, 2015.

[23] J. Wang, Z. Zhang, F. Xia, W. Yuan, and S. Lee, "An energy efficient stable election-based routing algorithm for wireless sensor networks," Sensors, vol. 13, no. 11, pp. 14301-14320, 2013.

[24] S. Naeimi, H. Ghafghazi, C.-O. Chow, and H. Ishii, "A survey on the taxonomy of cluster-based routing protocols for homogeneous wireless sensor networks," Sensors, vol. 12, no. 6, pp. 7350-7409, 2012.

[25] N. Javaid, M. Aslam, A. Ahmad, Z. A. Khan, and T. A. Alghamdi, "MCEEC: multi-hop centralized energy efficient clustering routing protocol for WSNs," in Proceedings of the 2014 1st IEEE International Conference on Communications (ICC '14), pp. 1784-1789, June 2014.

[26] M. Dong, K. Ota, and A. Liu, "RMER: reliable and energyefficient data collection for large-scale wireless sensor networks," IEEE Internet of Things Journal, vol. 3, no. 4, pp. 511-519, 2016.

[27] A. I. Al-Sulaifanie, S. Biswas, and B. K. Al-Sulaifanie, "AHMAC: adaptive hierarchical MAC protocol for low-rate wireless sensor network applications," Journal of Sensors, vol. 2017, Article ID 8105954, 18 pages, 2017. 


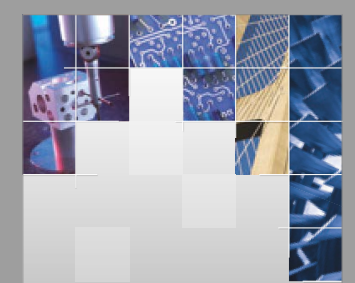

\section{Enfincering}
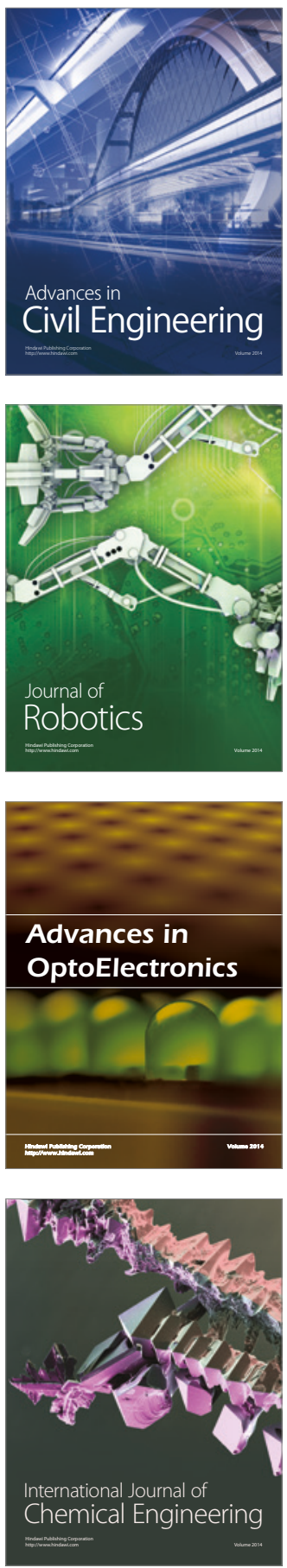

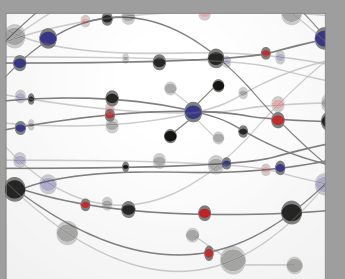

The Scientific World Journal

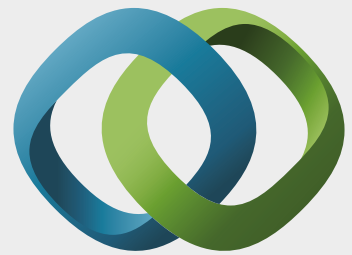

\section{Hindawi}

Submit your manuscripts at

https://www.hindawi.com
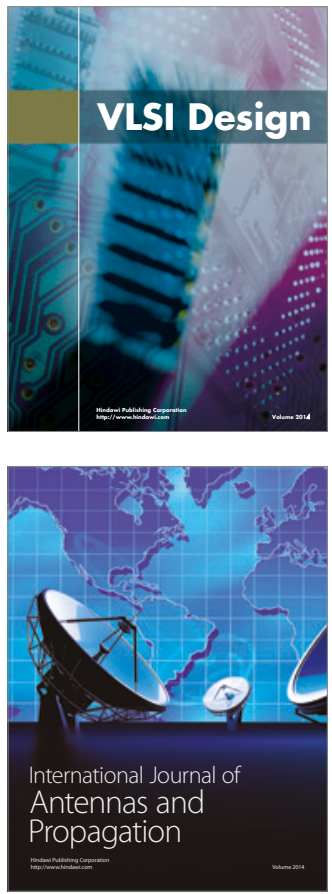

\section{Rotating}

Machinery
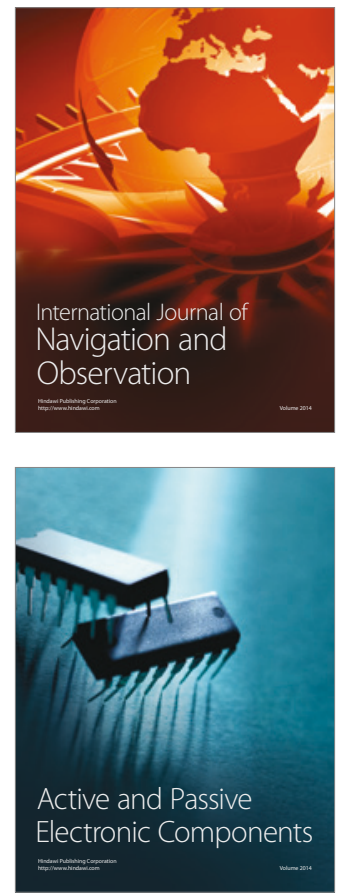
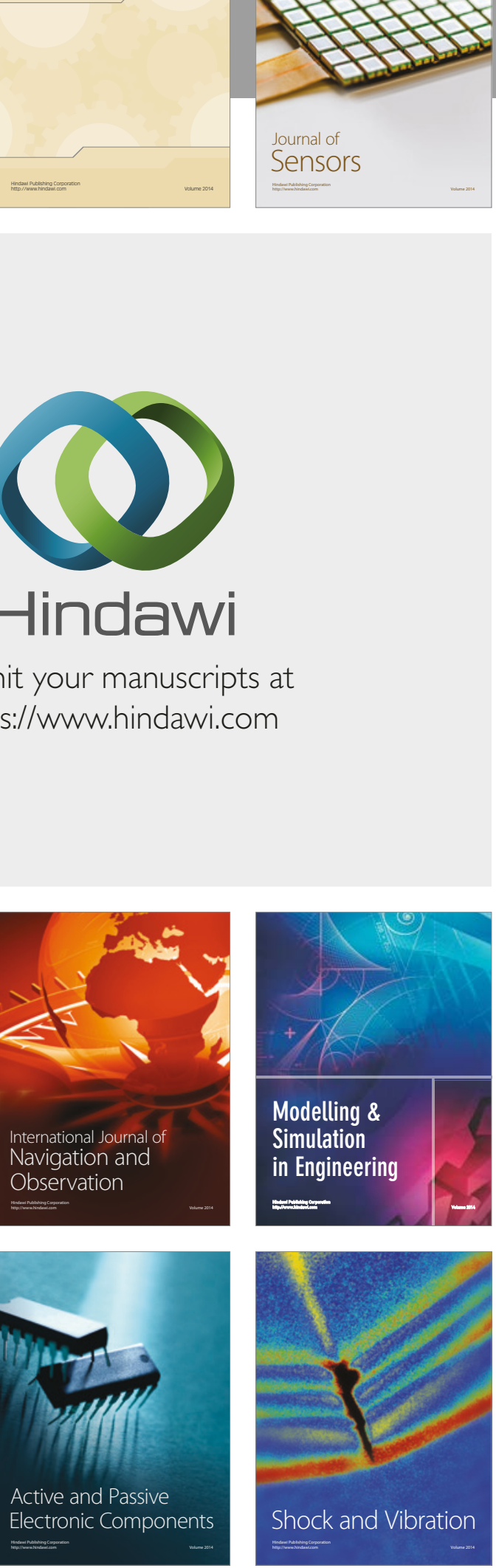
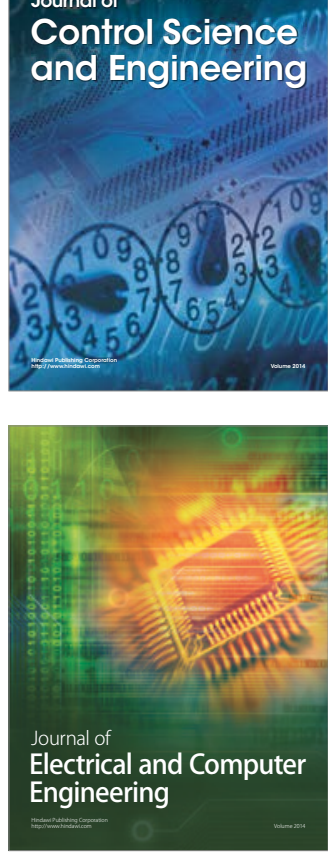

Distributed

Journal of

Control Science

and Engineering
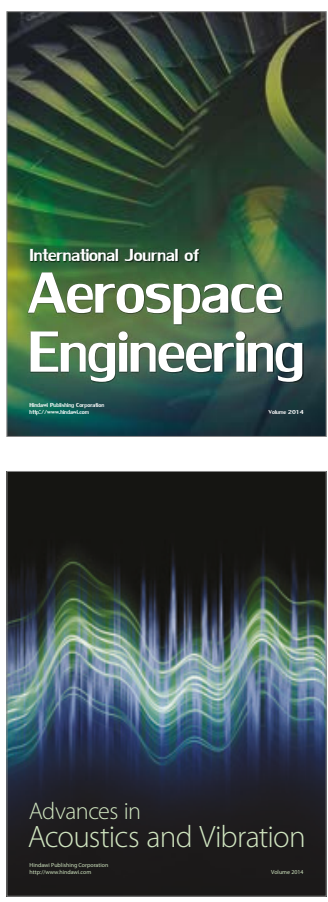

Sensor Networks 